\title{
Circular dichroism in photoelectron angular distributions as a probe of atomic and molecular alignment
}

\author{
Richard L. Dubs, S. N. Dixit, and V. McKoy \\ Arthur Amos Noyes Laboratory of Chemical Physics, ") California Institute of Technology, Pasadena, \\ California 91125
}

(Received 24 December 1985; accepted 4 April 1986)

\begin{abstract}
In this paper we show that circular dichroism in photoelectron angular distributions (CDAD) can be used to probe atomic and molecular alignment in the gas phase. Careful choice of photon (left or right circularly polarized) propagation and photoelectron collection directions breaks the cylindrical symmetry of the target, giving rise to dichroic effects. CDAD exists in the electric dipole approximation. We illustrate the sensitivity of CDAD to alignment by considering photoionization of the $A^{2} \Sigma^{+}$state of NO. Most of the cases of alignment we consider are created by multiphoton absorption while the others, more general, might be created in fragmentation, desorption, etc. The alignment created by $n$-photon absorption quickly reaches a classical limit which is reflected in the CDAD spectrum. Finally, we show that CDAD is also a sensitive probe of gas phase atomic state alignment by considering photoionization of the $7 P_{3 / 2}$ state of cesium created by single photon absorption from the ground state.
\end{abstract}

\section{INTRODUCTION}

In recent years, studies of orientational effects in chemical processes have attracted much attention due to the detailed dynamical information these studies can provide. Examples of such studies include atom-diatom collisions, ${ }^{1}$ unimolecular processes, ${ }^{2}$ electron stimulated desorption of adsorbed molecules, ${ }^{3}$ and molecular scattering from surfaces. ${ }^{4}$ Preparation of reactants with well characterized alignment and subsequent detection of product alignment/ orientation are integral parts of such investigations. The methods for creating aligned species include electronic ${ }^{5}$ or atomic ${ }^{6}$ impact, application of external electric and magnetic fields, ${ }^{7}$ photofragmentation, ${ }^{8,9}$ and photoabsorption. ${ }^{10-12}$ The detection of product alignment, on the other hand, has commonly been accomplished by measuring the fluorescence polarization $^{9(a)}$ resulting from either direct spontaneous emission or laser induced fluorescence.

In this paper we propose a new method for probing the alignment in atomic and molecular systems; namely, measuring the circular dichroism in photoelectron angular distributions (CDAD). CDAD spectra are obtained by taking the difference between angle-resolved photoelectron spectra (ARPES) for left and right circularly polarized light. Historically, circular dichroism (CD) has been associated with chiral molecules which lack a plane of symmetry and an inversion center. ${ }^{13-16}$ However, recently we have shown that CD can exist in photoelectron angular distributions from photoionization of oriented linear molecules ${ }^{17}$ and adsorbed atoms. ${ }^{18}$ Though previous studies of $\mathrm{CDAD}^{14,15,19,20}$ assumed strong spin-orbit coupling or high multipole interactions, our analyses ${ }^{17,18}$ showed that a proper choice of electron collection and the photon propagation direction can break the cylindrical symmetry of the target and give rise to dichroic effects in the electric-dipole approximation. We have also pointed out that CDAD studies often provide in-

\footnotetext{
a) Contribution No. 7339 .
}

formation complementary to that obtained from the ARPES studies. In this paper, we demonstrate that CDAD can also be used as a probe of the alignment of a gas phase target state. Since CDAD cannot exist in an isotropic target for symmetry reasons, the very existence of CDAD, therefore, implies alignment of the target. In addition, the shape of the CDAD spectrum provides details about this alignment.

The state of an atom or a molecule with total angular momentum $J$ is said to be aligned if the population of the $M_{J}$ magnetic sublevels is nonuniform. Alignment is differentiated from orientation in that the former requires the populations of $M_{J}$ and $-M_{J}$ states to be equal. Absorption of a linearly polarized photon from an isotropic initial state creates an aligned state whereas absorption of a circularly polarized photon gives rise to an oriented state. In this paper, we shall use the term "alignment" loosely to include orientation, the actual anisotropy being described by the populations of the $M_{J}$ levels. CDAD exists from aligned as well as oriented states and, as we show, is very sensitive to the details of the alignment.

In this paper, we shall discuss CDAD arising from photoionization of the $A^{2} \Sigma^{+}$state of NO. We shall consider alignment created in this $A^{2} \Sigma^{+}$state by mulitphoton absorption and will also choose some general alignments to illustrate the variation of CDAD with alignment. Finally, we examine CDAD in the photoionization of the $7 P_{3 / 2}$ state of Cs created by a single photon excitation from the ground $6 S_{1 / 2}$ state. It should be emphasized that while Parzynski's analysis ${ }^{20}$ of CDAD from atoms required strong spin-orbit coupling, this coupling is not necessary for the existence of CDAD. We include this coupling in our calculations, however, since it is present in the cesium atom.

Finally, a pump-probe type experiment can be designed as follows to observe CDAD in gas-phase atomic and molecular systems: Step 1: (creation of alignment); $n$-linearly polarized photons resonantly excite the isotropic initial state of atoms or molecules. The polarization vector of these pho- 
tons defines the laboratory frame $z$ axis. Step 2: (measurment of the alignment); the excited state is photoionized by a second laser with either left or right circular polarization and co-propagating with the first laser beam. Photoelectron angular distributions are then measured in the plane perpendicular to the photon propagation direction by rotating the direction of linear polarization. By delaying the probe beam relative to the pump, CDAD can also be used to monitor the evolution of alignment due to collisions. ${ }^{9(b)}$

\section{THEORY}

In this section we present the theory related to using CDAD as a probe of alignment. The analysis can be separated into a part dealing with the calculation of the anisotropy (alignment) in the atomic or molecular state and a part discussing CDAD from this aligned state. For the sake of simplicity we shall only consider alignment resulting from photoabsorption. Alignment created by other methods mentioned in the previous section can be calculated in an appropriate manner. Likewise, we will assume that the aligned state is ionized by a single left or right circularly polarized photon. Generalization to multiphoton ionization out of the aligned state can be carried out with a few additional steps.

\section{Alignment of the exclted state by multiphoton absorption}

Consider an excited state alignment created by absorption of a single photon, linearly polarized along the $z$ axis of the laboratory frame. In this case, the electric dipole moment operator can be written as

$$
D_{0}=\left(\frac{4 \pi}{3}\right)^{1 / 2} \sum_{s} r_{s} Y_{10}\left(\hat{r}_{s}\right),
$$

where $r_{s}$ is the magnitude of the position vector of the $s^{\text {th }}$ electron and $\hat{r}_{s}$ is the position of this electron in the laboratory frame. For a transition from an isotropic initial state with total angular momentum $J_{0}$ (all $M_{J_{0}}$ states are equally populated) to an intermediate state with angular momentum $J$, the alignment in the intermediate state, described by the population of various $M_{j}$ sublevels $\rho_{J, M_{j}}$, can be written as ${ }^{10}$

$$
\begin{aligned}
\rho_{J, M_{J}} & \propto \sum_{M_{J_{0}}}\left|\left\langle J M_{J}\left|D_{0}\right| J_{0} M_{J_{0}}\right\rangle\right|^{2}, \\
\propto & \left\langle J_{0} 1 M_{J_{0}} 0 \mid J M_{J}\right\rangle^{2} .
\end{aligned}
$$

In Eq. (2b), $\left\langle J_{0} 1 M_{J_{0}} 0 \mid J M_{J}\right\rangle$ denotes a Clebsch-Gordan coefficient. For a $n$-photon absorption from a $\left|J_{0} M_{J_{0}}\right\rangle$ state to $\left|J M_{J}\right\rangle, \rho_{J M_{J}}$ is similarly written as ${ }^{11}$

$\rho_{J M_{J}} \propto \sum_{M_{J_{0}}}\left|\sum_{\substack{J_{k} M_{J_{k}} \\ k=1, n-1}} \frac{\left\langle J M_{J}\left|D_{0}\right| J_{n-1} M_{J_{n-1}}\right\rangle\left\langle J_{n-1} M_{J_{n-1}}\left|D_{0}\right| J_{n-2} M_{J_{n-2}}\right\rangle \cdots\left\langle J_{1} M_{J_{1}}\left|D_{0}\right| J_{0} M_{J_{0}}\right\rangle}{\left(E_{0}-E_{1}+\hbar \omega\right)\left(E_{0}-E_{2}+2 \hbar \omega\right) \cdots\left[E_{0}-E_{n-1}+(n-1) \hbar \omega\right]}\right|^{2}$,

where $\hbar \omega$ is the photon energy and $E_{k}$ 's denote the energy of the $\left|J_{k} M_{J_{k}}\right\rangle$ state. Both Eqs. (2) and (3) are valid under weak field excitation conditions. For clarity, we have suppressed all other indices needed for the designation of various states. Note that $\rho_{J M_{J}}$, given by Eqs. (2) and (3), satisfy $\rho_{J M_{J}}=\rho_{J-M_{J}}$, i.e., the state $J$ is aligned and not oriented. For one-photon absorption, relative values of $\rho_{J M_{J}}$ depend only on the Clebsch-Gordan coefficients while for an $n$-photon $(n>1)$ absorption, even the relative values of $\rho_{J M}$ depend on the particular atomic or molecular system. Only in special cases where $J=J_{0} \pm n$ can the relative populations be represented purely by Clebsch-Gordan coefficients.

\section{Probing the alignment using CDAD}

In this subsection we shall discuss $C D A D$ in photoionization out of an aligned state. If $\left(d^{2} \sigma / d \Omega_{\hat{n}} d \Omega_{\hat{k}}\right)\left(J M_{J}\right)$ denotes the differential photoionization cross section (DCS) for the state $\left|J M_{J}\right\rangle$ then the total ( $m$-averaged) DCS out of the excited state is, ${ }^{11}$

$$
\frac{d^{2} \sigma}{d \Omega_{\hat{n}} d \Omega_{\hat{k}}}=\sum_{M_{J}} \rho_{J M_{J}} \frac{d^{2} \sigma}{d \Omega_{\hat{\AA}} d \Omega_{k}}\left(J M_{J}\right),
$$

where $\rho_{J M_{J}}$ are populations in the states $\left|J M_{J}\right\rangle$ that characterize the alignment. The CDAD spectra are obtained by taking the difference in the DCS's from Eq. (4) for left and right circularly polarized light. The $m$-resolved DCS $\left(d^{2} \sigma / d \Omega_{A} d \Omega_{\hat{k}}\right)\left(J M_{J}\right)$ is proportional to the square of the photoelectron matrix element $I_{k, \AA}^{J M_{s, \mu_{0}}}$ written as ${ }^{21}$

$$
I_{\mathbf{k}, \AA}^{J M_{j, \mu_{0}}}=\left\langle\psi_{f, \mathbf{k}}^{(-)}\left|D_{\mu_{0}}\right| \psi_{J M_{J}}\right\rangle
$$

In the above equation, $\mu_{0}$ denotes the polarization index ( 0 for linear and \pm 1 for circular polarization) in the ionizing photon frame, and $\left|\psi_{J M_{J}}\right\rangle$ and $\left|\psi_{f, \mathbf{k}}^{(-)}\right\rangle$the initial and final states, respectively. In the following, we will describe the calculation of this bound-free matrix element for photoionization of the $A^{2} \Sigma^{+}$state of NO and of the $7 P_{3 / 2}$ state of Cs.

\section{Molecules}

For the $A^{2} \Sigma^{+}$state of NO, the wave function $\left|\psi_{J_{M_{3}}}\right\rangle$ can be described in Hund's case (b) as ${ }^{22}$

$$
\begin{aligned}
\psi_{J M_{J}} \equiv & \left|\gamma \Lambda \mathrm{N} S J M_{J}\right\rangle=\sqrt{\frac{2 N+1}{8 \pi^{2}}} \sum_{M, M_{S}}\left\langle N S M M_{S} \mid J M_{J}\right\rangle \\
& \times \psi_{\gamma \Lambda}^{(e)}\left(\left\{\mathrm{r}_{i}^{\prime}\right\} ; R\right) D_{\Lambda M}^{N}\left(\hat{R}^{\prime}\right) \chi_{\nu}(R)\left|S M_{S}\right\rangle .
\end{aligned}
$$

Here primed coordinates refer to the molecular frame while unprimed coordinates refer to the laboratory frame. $\psi_{\gamma \Lambda}^{(e)}$ is the electronic wave function that depends on the internuclear separation, $R, \chi_{v}(R)$ is the wave function for the vibrational state $v$, and $\left|S M_{S}\right\rangle$ is the spin wave function. $D_{\Lambda M}^{N}\left(\hat{R}^{\prime}\right)$ denotes the symmetric top rotational wave function with total (rotational plus electronic) angular momentum $N$, the projection of this angular momentum along the $z$ axis of the molecular frame being $\Lambda(\Lambda=0$ for $\Sigma$ states, \pm 1 for $\Pi$ states, etc.), and the projection along the $z$ axis of the 
laboratory frame being $M$. The notation of $\operatorname{Ros}^{23}$ is used throughout. $\widehat{R}$ ' refers to the coordinates of the laboratory $z$ axis in the molecular frame. The factor $\sqrt{(2 N+1) / 8 \pi^{2}}$ ensures normalization of the total wave function. $\gamma$ contains all other subscripts necessary for an unambiguous designation of the state.

The continuum function $\psi_{f, \mathbf{k}}^{(-)}$is a product of wave functions for the photoelectron $|\phi\rangle$ and the resulting molecular ion $\left|\gamma_{+} \Lambda_{+} N_{+} S_{+} J_{+} M_{J_{+}}\right\rangle$:

$$
\psi_{f, \mathbf{k}}^{(-)}=|\phi\rangle\left|\gamma_{+} \Lambda_{+} N_{+} S_{+} J_{+} M_{J_{+}}\right\rangle
$$

We calculate $\psi_{f, \mathbf{k}}^{(-)}$using the iterative Schwinger variational technique. ${ }^{21}$ The electronic wave function for the continuum electron can be expanded in partial waves ${ }^{11}$ :

$|\phi\rangle=\left|\frac{1}{2} m_{\sigma}\right\rangle \sum_{m \lambda} i^{i} e^{-i \eta_{\mu}} Y_{l m}^{*}(\hat{k}) D_{\lambda m}^{l}\left(\hat{R}^{\prime}\right) \psi_{k l \lambda}\left(\left\{\mathbf{r}^{\prime}\right\} ; R\right)$.

$m_{\sigma}$ is the projection of the electron spin on the laboratory frame $z$ axis. $k$ denotes the photoelectron momentum and $k$ its direction in the laboratory frame. $\operatorname{lm} \lambda$ denote, respective$1 y$, the angular momentum of the electron and its projections along the laboratory and molecular $z$ axes. The ionic wave function can be written as

$$
\begin{aligned}
& \left|\lambda_{+} \Lambda_{+} N_{+} S_{+} J_{+} M_{J_{+}}\right\rangle \\
& =\sqrt{\frac{2 N_{+}+1}{8 \pi^{2}}} \sum_{M_{+} M_{S_{+}}}\left\langle N_{+} S_{+} M_{+} M_{S_{+}} \mid J_{+} M_{J_{+}}\right\rangle \\
& \quad \times \psi_{\gamma_{+}(e)}^{(e)}\left(\left\{\mathbf{r}_{i}^{\prime}\right\} ; R\right) \chi_{\nu_{+}}\left|S_{+} M_{S_{+}}\right\rangle D_{\Lambda_{+} M_{+}}^{N_{+}}\left(\hat{R}^{\prime}\right),
\end{aligned}
$$

where $v_{+}, J_{+}, M_{J_{+}}$, and $\Lambda_{+}$denote, respectively, the ionic vibrational quantum number, the total angular momentum quantum number, and the projection of $J_{+}$on the laboratory and molecular $z$ axes. $S_{+}$is the total spin of the ion and $M_{S_{+}}$ its projection on the laboratory $z$ axis.

In the single particle picture, $D_{\mu_{0}}$ of Eq. (5) can be written in the frame of the ionizing photon as

$$
D_{\mu_{0}}=(4 \pi / 3)^{1 / 2} r Y_{1 \mu_{0}}(\hat{r})
$$

As the coordinate frames of the exciting and ionizing photons need not coincide, we must transform $D_{\mu_{0}}$ into the frame of the exciting photon. $D_{\mu_{\mathrm{o}}}$ can then be written as

$$
D_{\mu_{0}}=\left(\frac{4 \pi}{3}\right)^{1 / 2} r \sum_{\mu} D_{\mu \mu_{0}}^{1}\left(\hat{R}^{\prime \prime}\right) \sum_{\mu^{*}} D_{\mu^{\mu} \mu}^{1}\left(\hat{R}^{\prime}\right) Y_{1 \mu^{\prime \prime}}\left(\hat{r}^{\prime}\right) \text {. }
$$

Here, $\widehat{R}$ " specifies the coordinates of the $z$ axis of the ionizing photon frame in the laboratory frame. $\hat{r}^{\prime}$ is the coordinate of the position vector in the molecular frame.

Substituting Eqs. (6), (7), (8), (9), and (11) into Eq. (5) and integrating over molecular orientations, Eq. (5) becomes

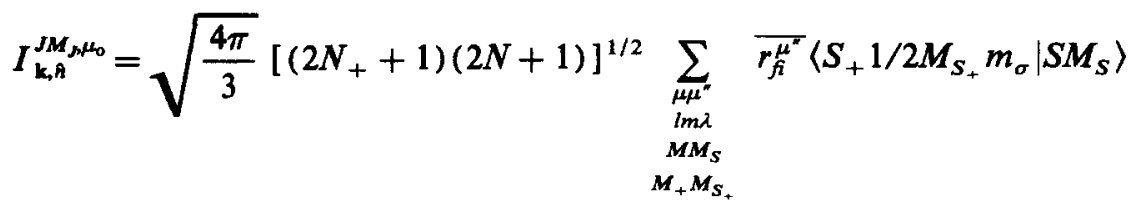

$$
\begin{aligned}
& \times D_{\mu \mu_{0}}^{1}\left(\hat{R}^{n}\right)\left\langle N_{+} S_{+} M_{+} M_{S_{+}} \mid J_{+} M_{J_{+}}\right\rangle\left\langle N S M M_{S} \mid J M_{J}\right\rangle \\
& \times(-i)^{b} e^{i \eta_{u}} Y_{l m}(\hat{k})(-1)^{\mu-\mu^{*}+\Lambda_{+}-M_{+}} \sum_{N_{t}} \frac{1}{2 N_{t}+1}\left\langle l 1-\lambda \mu^{\prime \prime} \mid N_{t} \mu^{\prime \prime}-\lambda\right\rangle\left\langle l 1-m \mu \mid N_{t} \mu-m\right\rangle \\
& \times\left\langle N N_{+} M-M_{+} \mid N_{t} m-\mu\right\rangle\left\langle N N_{+} \Lambda-\Lambda_{+} \mid N_{t} \lambda-\mu^{\prime \prime}\right\rangle,
\end{aligned}
$$

where

$$
\overline{r_{f}^{\left(\mu^{*}\right)}}=\int \chi_{v_{+}}(R) r_{f}^{\left(\mu^{*}\right)} \chi_{v}(R) d R
$$

and

$$
r_{f^{*}}^{\left(\mu^{*}\right)}=\left\langle\psi_{k l \lambda}\left(\left\{\mathbf{r}_{i}^{\prime}\right\} ; R\right) \psi_{\gamma_{+} \Lambda_{+}}^{(e)}\left(\left\{\mathbf{r}_{i}^{\prime}\right\} ; R\right)\left|r Y_{1 \mu^{-}}\left(\hat{r}^{\prime}\right)\right| \psi_{\gamma \Lambda}^{e}\left(\left\{\mathbf{r}_{i}^{\prime}\right\} ; R\right)\right\rangle
$$

denotes the photoionization electronic transition moment.

Using Eq. (12), Eq. (4) can be written as

$$
\frac{d^{2} \sigma}{d \Omega_{\hat{k}} d \Omega_{\hat{k}}}=(-1)^{\mu_{0}} \sum_{M_{J}} \rho_{J M_{J}} \sum_{L L^{\prime} M^{\prime}}\left\langle 11-\mu_{0} \mu_{0} \mid L^{\prime} 0\right\rangle \beta_{L L^{\prime} M^{\prime}}^{M_{J}} Y_{L-M^{\prime}}\left(\theta_{k}, \phi_{k}\right) Y_{L^{\prime} M^{\prime}}\left(\theta_{p}, \phi_{p}\right),
$$

where

$$
\begin{aligned}
\beta_{L L^{\prime} M^{\prime}}^{M_{J}}= & {\left[(2 L+1)\left(L^{\prime}+1\right)\right]^{-1 / 2} \sum_{\substack{m_{\sigma} \\
N_{+} J_{+} M_{J_{+}}}} \sum_{l m m^{\prime} \mu^{\prime}} I_{l m \mu}^{a} I_{l^{\prime} m^{\prime} \mu^{\prime}}^{\alpha^{*}}(-1)^{m^{\prime}-\mu} } \\
& \times\left[(2 l+1)\left(2 l^{\prime}+1\right)\right]^{1 / 2}\left\langle l l^{\prime} 00 \mid L 0\right\rangle\left\langle l l^{\prime}-m m^{\prime} \mid L M^{\prime}\right\rangle\left\langle 11-\mu \mu^{\prime} \mid L^{\prime} M^{\prime}\right\rangle
\end{aligned}
$$

and 


$$
\begin{aligned}
I_{l m \mu}^{\alpha}= & {\left[\left(2 N_{+}+1\right)(2 N+1)\right]^{1 / 2} \sqrt{\frac{4 \pi}{3}}(-i)^{l}(-1)^{\Lambda_{+}-M_{+}} \sum_{\substack{\mu^{\prime \prime \lambda} \\
M_{+} M_{S_{+}}}}(-1)^{\mu-\mu^{\prime \prime}} e^{i \eta_{\mu}} \overline{r\left(\mu^{\prime}\right)}\left\langle S_{+} 1 / 2 M_{S_{+}} m_{\sigma} \mid S M_{S}\right\rangle } \\
& \times\left\langle N S M M_{S} \mid J M_{J}\right\rangle\left\langle N_{+} S_{+} M_{+} M_{S_{+}} \mid J_{+} M_{J_{+}}\right\rangle \sum_{N_{t}} \frac{1}{\left(2 N_{t}+1\right)}\left\langle l 1-\lambda \mu^{\prime \prime} \mid N_{t} \mu^{\prime \prime}-\lambda\right\rangle \\
& \times\left\langle l 1-m \mu \mid N_{t} \mu-m\right\rangle\left\langle N N_{+} \Lambda-\Lambda_{+} \mid N_{t} \lambda-\mu^{\prime \prime}\right\rangle\left\langle N N_{+} M-M_{+} \mid N_{t} m-\mu\right\rangle
\end{aligned}
$$

In the above equations, $\alpha$ refers to the variables $m_{\sigma}, N_{+}, J_{+}, M_{J_{+}}$, and $\left(\theta_{k}, \phi_{k}\right)$ and $\left(\theta_{p}, \phi_{p}\right)$ denote, respectively, the collection angle of the photoelectron and the propagation direction of the circularly polarized ionizing photon in the laboratory frame.

Equation (15) makes calculation of ARPES spectra simple. The $\beta_{L L}^{M_{J} M^{\prime}}$ are calculated once for a given photon energy, after which Eq. (15) is used to quickly calculate spectra for any alignment, light polarization, or experimental configuration of photon propagation direction or electron collection direction.

The CDAD signal is defined as the difference in the differential cross section of Eq. (14) for left and right circularly polarized light. ${ }^{17}$

$$
\frac{d \sigma^{L-R}}{d \Omega_{\hat{k}} d \Omega_{\AA}}=\frac{d \sigma^{+1}}{d \Omega_{\hat{k}} d \Omega_{\mathfrak{n}}}-\frac{d \sigma^{-1}}{d \Omega_{\hat{k}} d \Omega_{\mathfrak{n}}} .
$$

In Eq. (15), only $(-1)^{\mu_{0}}\left\langle 11-\mu_{0} \mu_{0} \mid L^{\prime} 0\right\rangle$ depends on the polarization of the photon. Thus, due to the symmetry property

$$
\left\langle 11 \mu_{0}-\mu_{0} \mid L^{\prime} 0\right\rangle=(-1)^{L^{\prime}}\left\langle 11-\mu_{0} \mu_{0} \mid L^{\prime} 0\right\rangle,
$$

only terms in which $L^{\prime}=1$ will contribute to CDAD. In addition, due to the symmetry properties of $I_{l m \mu, M}^{\alpha}$, only terms for which $M^{\prime}= \pm 1$ will contribute to CDAD and the corresponding $\beta_{L 1 \pm 1}$ will be pure imaginary numbers. ${ }^{17}$

\section{Atoms}

For atoms, the bound-continuum matrix element is still defined by Eq. (14) where ${ }^{24}$

$$
\begin{aligned}
\left|\psi_{f, k^{\prime}}^{(-)}\right\rangle= & 4 \pi\left[\frac{\pi}{2 k}\right]^{1 / 2} \sum_{l=0}^{\infty} \sum_{m^{\prime}=-l^{\prime}}^{l^{\prime}} i^{l^{\prime}} e^{-i \delta_{l^{\prime}}} Y_{l^{\prime} m^{\prime}}^{*}(\hat{k}) \\
& \times \sum_{j^{\prime} m_{j}^{\prime}} R_{E l^{\prime} J^{\prime}}(r)\left\langle l^{\prime} \frac{1}{2} m^{\prime} m_{\sigma} \mid J^{\prime} m_{J}^{\prime}\right\rangle\left|l_{2} J^{\prime} m_{J}^{\prime}\right\rangle,
\end{aligned}
$$

$$
\left|\psi_{i}^{J M_{J}}\right\rangle=R_{n L J}(r)\left|l \frac{1}{2} J m_{J}\right\rangle
$$

and

$$
\left|l \frac{1}{2} J m_{J}\right\rangle=\sum_{m} \sum_{m_{\sigma}}\left\langle l \frac{1}{2} m m_{\sigma} \mid J m_{J}\right\rangle Y_{l m}(\hat{r})\left|\frac{1}{2} m_{\sigma}\right\rangle .
$$

Both the radial wave functions, $R_{E L}(r)$ and the phase shifts $\delta_{l}$ are calculated as in Ref. 24. $\hat{r}$ and $\hat{k}$ are expressed in the laboratory frame. The dipole moment operator $D_{\mu_{0}}$, expressed in Eq. (10), is rewritten as

$$
D_{\mu_{0}}=\left(\frac{4 \pi}{3}\right)^{1 / 2} r \sum_{\mu} D_{\mu \mu_{0}}^{1}\left(R^{\prime \prime}\right) Y_{l \mu}(\hat{r})
$$

Substituting Eqs. (20)-(23) into Eq. (5), Eqs. (15) and (16) are again obtained, with the sums over $K_{+}, J_{+}$, and $M_{+}$omitted and $\alpha=m_{\sigma}$ in Eq. (16). Now $I_{l m u}^{a}$ is defined as

$$
\begin{aligned}
& I_{l^{\prime} m^{\prime} u^{\prime}}^{\alpha}=(-i)^{l^{\prime}} e^{i \delta_{t}} \overline{r_{f i}}(-1)^{J+l+1 / 2} \\
& \times \sqrt{\frac{3}{4 \pi}}[(2 J+1)(2 l+1)]^{1 / 2} \\
& \times \sum_{J^{\prime} m_{J}^{\prime}}\left\langle l^{\prime} \frac{1}{2} m^{\prime} m_{\sigma} \mid J^{\prime} m_{J}^{\prime}\right\rangle\left\{\begin{array}{ccc}
l^{\prime} & J^{\prime} & \frac{1}{2} \\
J & l & 1
\end{array}\right\} \\
& \times\left\langle J 1 m_{J} u^{\prime} \mid J^{\prime} m_{J}^{\prime}\right\rangle\left\langle l 100 \mid l^{\prime} 0\right\rangle,
\end{aligned}
$$

where

$$
\overline{r_{f i}}=\left\langle R_{E l^{\prime} j^{\prime}}(r)|r| R_{n l j}(r)\right\rangle
$$

\section{RESULTS AND DISCUSSION}

\section{Molecules}

In this section we present the results for CDAD in photoionization of the $A^{2} \Sigma^{+}$state of NO. The ionizing photon wavelength is chosen to be about $225 \mathrm{~nm}$. The bound-free matrix elements were taken from the calculations presented in Ref. 22. In Fig. 1, we present ARPES spectra for left and right polarized light and the resulting CDAD spectra. Figure 1 (a) corresponds to single photon $J_{0}=1 / 2 \rightarrow J=3 / 2$ excitation to the $A$ state while Fig. 1(b) corresponds to $J_{0}=3 / 2 \rightarrow J=5 / 2$ excitation. The relative populations $\rho_{J M_{J}}$ are given in Table $I$ (cases $A$ and $B$ ). Both CDAD spectra have a $\sin 2 \theta_{k}$ dependence. This is a reflection of a one-photon absorption alignment. Mathematically, the $\sin 2 \theta_{k}$ dependence arises because only $\beta_{21 \pm 1}$ contribute to CDAD in this case. At $\theta_{k}=45^{\circ} \mathrm{CDAD}$ spectra are about $15 \%$ of ARPES spectra. The relative strengths of CDAD and ARPES spectra depend on specific values of molecular parameters and could be different for other systems.

In Figs. 2(a) - 2(c) we present CDAD and ARPES spectra for individual $M_{J}(1 / 2,3 / 2$, and $5 / 2)$ states of the $A^{2} \Sigma^{+}(J=5 / 2)$ level. The relative populations are again given in Table I (cases C, D, and E). Case C corresponds to two-photon excitation from a $J_{0}=1 / 2$ state. Although the alignments described by cases $D$ and $E$ cannot be produced by multiphoton absorption of linearly polarized light, they might result from photofragmentation reactions, gas-solid scattering, desorption, etc. In addition, a state initially prepared in a $M_{J}= \pm 1 / 2$ state may evolve, e.g., by collision, into a state with a distribution of $M_{J}$ values. ${ }^{9(b)}$ In this case, the net CDAD spectrum will be a weighted sum of the spectra for individual $M_{J}$ values. Note that from an isotropic 


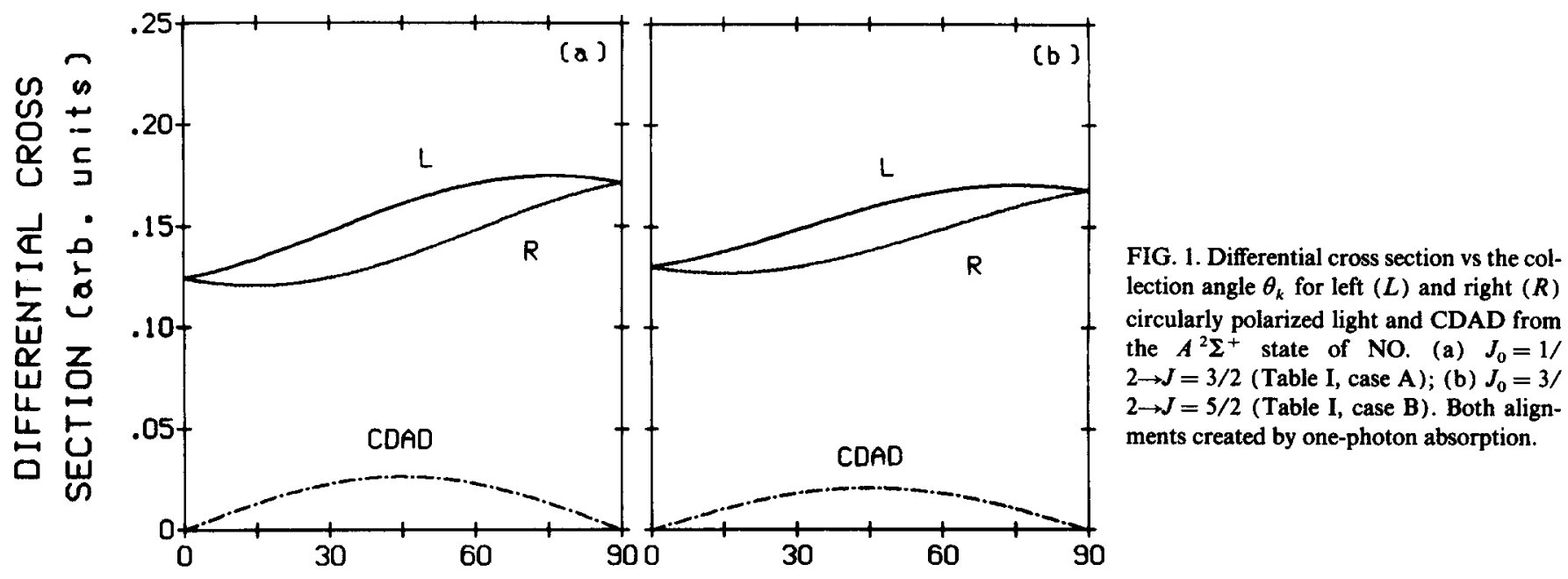

COLLECTION ANGLE $\theta_{k}$ (deg)

state (all $M_{J}$ 's equally populated), CDAD cannot exist for symmetry reasons. The sum of the CDAD spectra in Figs. $2(a), 2(b)$, and 2 (c) thus gives a spectrum of zero magnitude at all angles.

It is clear from these results that CDAD spectra are quite sensitive to the relative populations of the $M_{J}$ states. Comparison of one- and two-photon CDAD [Figs. 1(b) and 2(a) ] indicates that CDAD probes the anisotropy of the aligned excited state. While the single-photon excitation CDAD are proportional to $Y_{2 \pm 1}\left(\theta_{k}, \phi_{k}\right)$, the two-photon excitation CDAD can be expressed as a linear combination of $Y_{2 \pm 1}\left(\theta_{k}, \phi_{k}\right)$ and $Y_{4 \pm 1}\left(\theta_{k}, \phi_{k}\right)$, the relative weights of which depend on the particulars of the process. In fact, one can show (although the algebra is quite tedious) that CDAD spectra in photoionization from an $n$-photon excited state will contain terms $Y_{2 \pm 1}, \quad Y_{4 \pm 1}, \ldots$ up to $Y_{2 n, \pm 1}\left(\theta_{k}, \phi_{k}\right)$. The ARPES spectra, on the other hand, contain terms up to $Y_{2(n+1), m}\left(\theta_{k}, \phi_{k}\right)$. This difference is a consequence of the fact that CDAD spectra, each being a difference between two ARPES spectra, have lost the infor- mation about the final photoionization step and are therefore only sensitive to the alignment in the $n$-photon excited state. Mathematically, this result is a direct consequence of the fact that, for the ionizing photon, $L^{\prime}$ can only take the value of 1 for $\mathrm{CDAD}^{17}$ while it can have its maximum value of 2 for ARPES.

What happens to the CDAD spectra as more and more pump photons are absorbed? In Fig. 3, the ARPES and CDAD spectra are shown for photoionization from the $A$ state with the alignment created by the $J_{0}=1 / 2 \rightarrow J=11 / 2$ five-photon absorption from the $X$ state (case $\mathrm{F}$ in Table I). Note that the magnitude of the CDAD spectrum has increased slightly to $25 \%$ of the ARPES spectra, but otherwise appears very similar to that for the alignment created by two-photon absorption [Fig. 2(a)]. The CDAD spectrum for photoionization out of an aligned state prepared by the $J_{0}=1 / 2 \rightarrow J=21 / 2$ ten-photon absorption is found to be virtually identical to that in Fig. 3. Thus, the alignment of the molecule appears to quickly reach a limit after which absorption of additional photons has little effect.

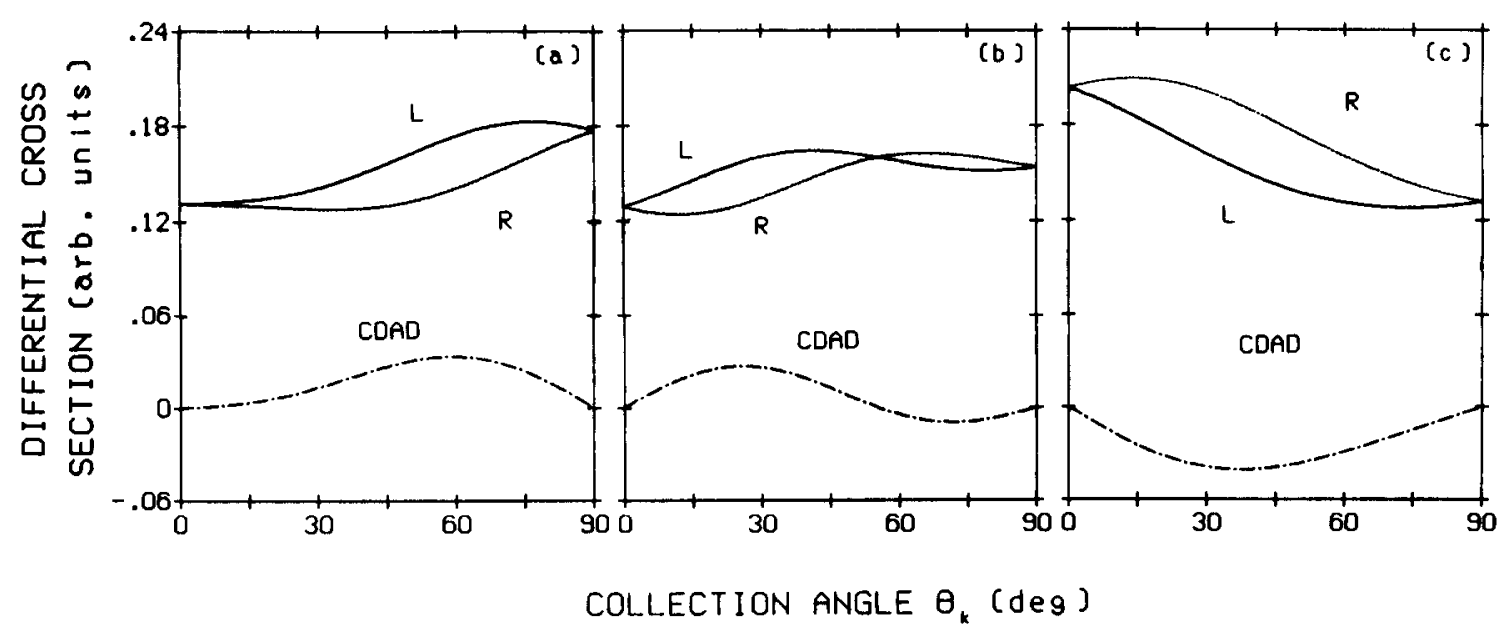

FIG. 2. Differential cross section vs the collection angle $\theta_{k}$ for left $(L)$ and right $(R)$ circularly polarized light and CDAD from the $A^{2} \Sigma^{+}$state of NO. For all cases $J=5 / 2$. (a) $M_{J}= \pm 1 / 2$ (Table I case C); (b) $M_{J}= \pm 3 / 2$ (Table I, case D); (c) $M_{J}= \pm 5 / 2$ (Table I, case E). Alignment for (a) created by two-photon absorption $J_{0}=1 / 2 \rightarrow J=5 / 2$. 
TABLE I. Relative populations $2 \rho_{J M_{J}}$ of $M_{J}$ states.

\begin{tabular}{|c|c|c|c|c|c|c|c|c|c|c|c|c|c|c|}
\hline Case & $J$ & $-11 / 2$ & $-9 / 2$ & $-7 / 2$ & $-5 / 2$ & $-3 / 2$ & $-1 / 2$ & $1 / 2$ & $3 / 2$ & $5 / 2$ & $7 / 2$ & $9 / 2$ & $11 / 2$ & Comment \\
\hline $\mathbf{A}$ & $3 / 2$ & & & & & 0 & 1 & 1 & 0 & & & & & One-photon $1 / 2 \rightarrow 3 / 2$ \\
\hline $\mathbf{B}$ & $5 / 2$ & & & & 0 & $2 / 5$ & $3 / 5$ & $3 / 5$ & $2 / 5$ & $\mathbf{0}$ & & & & One-photon $3 / 2 \rightarrow 5 / 2$ \\
\hline C & $5 / 2$ & & & & 0 & 0 & 1 & 1 & 0 & 0 & & & & Two-photon $1 / 2 \rightarrow 5 / 2$ \\
\hline D & $5 / 2$ & & & & 0 & 1 & 0 & 0 & 1 & 0 & & & & See the text \\
\hline $\mathbf{E}$ & $5 / 2$ & & & & 1 & 0 & 0 & 0 & 0 & 1 & & & & See the text \\
\hline$F$ & $11 / 2$ & 0 & 0 & 0 & 0 & 0 & 1 & 1 & 0 & 0 & 0 & $\mathbf{0}$ & 0 & Five-photon $1 / 2 \longrightarrow \longrightarrow \longrightarrow 11 / 2$ \\
\hline
\end{tabular}

\section{THE CLASSICAL PICTURE OF THE ALIGNMENT}

To understand the above limiting behavior, let us consider a rigid rotor in a $\left|J_{0} M_{0}\right\rangle$ state. If all the $M_{0}$ states are equally populated, the spatial distribution of the rotor axis is simply

$$
P(\theta, \phi)=\sum_{M_{0}} P_{J_{0} M_{0}}(\theta, \phi)=\left|Y_{00}\right|^{2}=\text { const. }
$$

In the absence of the spin-orbit coupling, absorption of $n$ linearly polarized photons will lead to a spatial distribution $\left|Y_{n 0}\right|^{2}$. Absorption of $n$-photons in molecules with spin-orbit coupling will give rise to spatial distributions involving $\left|Y_{n m}\right|^{2}, m \neq 0$ as well. For example, a two-photon transition from $|1 / 21 / 2\rangle \rightarrow|5 / 21 / 2\rangle$ in NO will lead to a spatial distribution

$$
\begin{aligned}
P_{5 / 21 / 2}(\theta, \phi)= & \langle 21 / 201 / 2 \mid 5 / 21 / 2\rangle^{2}\left|Y_{20}\right|^{2} \\
& +\langle 21 / 21-1 / 2 \mid 5 / 21 / 2\rangle^{2}\left|Y_{21}\right|^{2} .
\end{aligned}
$$

Similarly a five-photon $1 / 2 \rightarrow 11 / 2$ transition implies

$$
\begin{aligned}
P_{11 / 21 / 2}(\theta, \phi)= & \langle 51 / 201 / 2 \mid 11 / 21 / 2\rangle^{2}\left|Y_{50}\right|^{2} \\
& +\langle 51 / 21-1 / 2 \mid 11 / 21 / 2\rangle^{2}\left|Y_{51}\right|^{2} .
\end{aligned}
$$

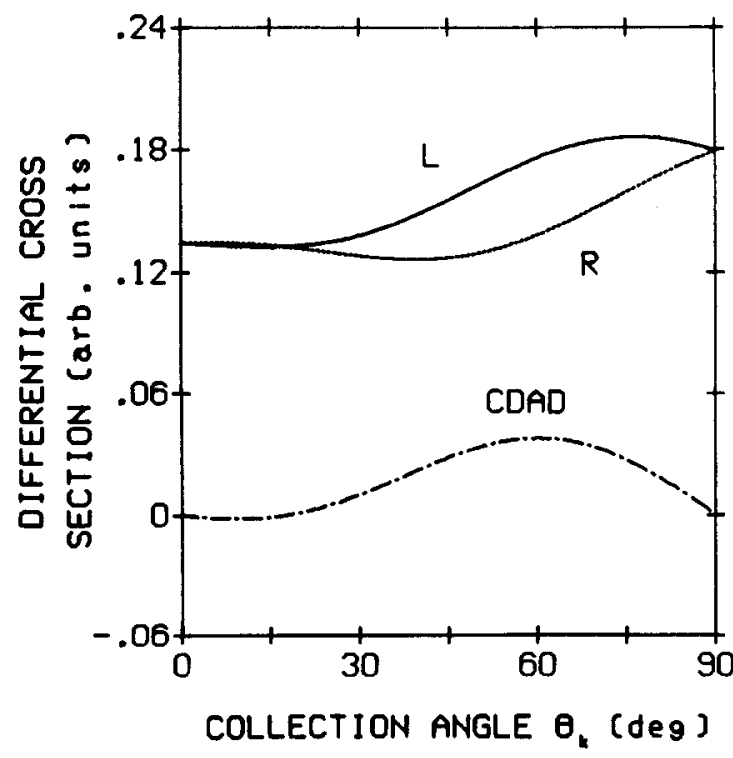

FIG. 3. Differential cross section vs the collection angle $\theta_{k}$ for left $(L)$ and right $(R)$ circularly polarized light and CDAD from the $A^{2} \Sigma^{+}$state of NO. Alignment created by five-photon absorption $J_{0}=1 / 2 \rightarrow J=11 / 2$ (Table I, case F).
Using the method described in Ref. 25, we have performed photoionization calculations from oriented NO molecules (ignoring spin) whose orientations in space are weighted by $\left|Y_{J M}\right|^{2}$. The results for $\left|Y_{20}(\theta, \phi)\right|^{2}$ and $\left|Y_{50}(\theta, \phi)\right|^{2}$ are shown in Figs. 4(a) and 4(b), respectively. Comparing these with fully quantum mechanical calculations [Figs. 2(a) and 3], we see that the classical calculation agrees well with the quantum mechanical one for five-photon absorption $\left(\left|Y_{50}\right|^{2}\right)$ while for two-photon absorption the agreement is poor. This discrepancy is not due to a diminishing distribution of $\left|Y_{J 1}\right|^{2}$ with increasing $J$ but rather due to the fact that the distributions $\left|Y_{J O}\right|^{2}$ and $\left|Y_{J 1}\right|^{2}$ become more and more similar for high $J$. In other words, $\left|Y_{50}\right|^{2}$ and $\left|Y_{51}\right|^{2}$ have similar spatial distributions whereas $\left|Y_{20}\right|^{2}$ and $\left|Y_{21}\right|^{2}$ have quite different ones. Thus as $J$ increases, $\left|Y_{J 0}\right|^{2}$ accurately describes the spatial orientation of the excited $A^{2} \Sigma^{+}$state. This statement is also a reflection of the decreasing importance of the $1 / 2$ unit spin at high $J$.

We are now in a position to understand our earlier observation that alignment of a molecule appears to quickly reach a limit after which absorption of additional photons has little effect. To examine the $\theta$ dependence of the alignment of a molecule after it has absorbed $J$ photons we look at

$$
\left|Y_{J 0}(\theta, \phi)\right|^{2} \sin \theta,
$$

where the $\sin \theta$ accounts for the increase in solid angle with increasing $\theta$. As $J$ increases, the $\left|Y_{J 0}(\theta, \phi)\right|^{2}$ oscillates more and more rapidly (with $J$ nodes from $\theta=0$ to $\theta=\pi$ ). If we replace this rapidly oscillating part by its average value, ${ }^{26}$ we find

$$
\left|Y_{J 0}(\theta, \phi)\right|^{2}=\frac{1}{2 \pi^{2} \sin \theta} \text { for } J \text { large and } \theta>\frac{1}{J} .
$$

Near the $z$ axis $\left|Y_{J 0}(\theta, \phi)\right|^{2}$ has a large finite value; however, this contribution is cancelled by the $\sin \theta$ in Eq. (29). Note that the right-hand side of Eq. (30) is independent of J. For this reason, after a few photons are absorbed, the alignment quickly reaches a limit. As we have shown, the CDAD spectra clearly reflect this fact.

\section{Atoms}

In Fig. 5, ARPES and CDAD spectra are shown for photoionization from the $7 P_{3 / 2}$ state of Cs. The alignment of the state is created by one-photon absorption from the $6 S_{1 / 2}$ state (Table I, case A). The energy of the ionizing photon is assumed to be equal to the energy difference between the $6 S_{1 / 2}$ and $7 P_{3 / 2}$ states. The required atomic parameters were calculated using the quantum-defect theory. We again note 


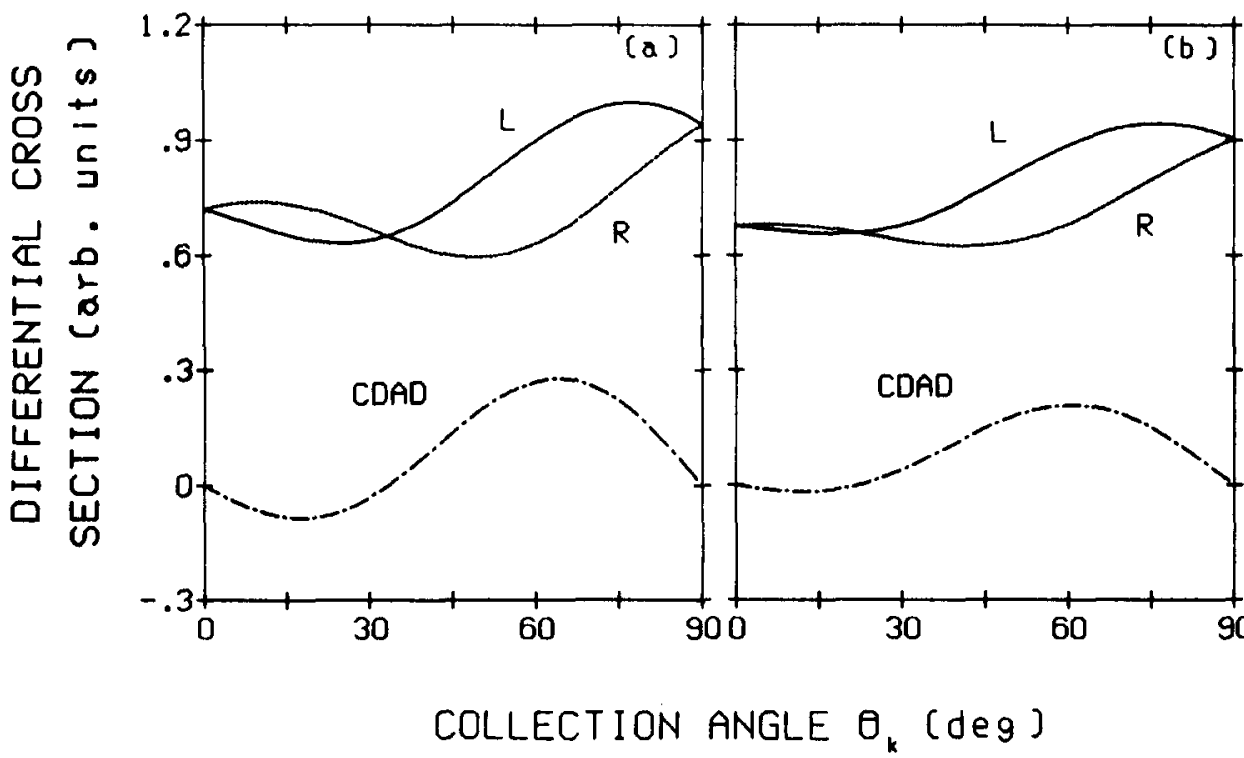

FIG. 4. Differential cross section vs the collection angle $\theta_{k}$ for left (L) and right (R) circularly polarized light and CDAD from the $A^{2} \Sigma^{+}$state of NO. Classical alignment ignoring spin: (a) $(J, M)=(2,0)$; (b) $(J, M)=(5,0)$.

the $\sin 2 \theta_{k}$ dependence of the CDAD spectrum, a signature of the one-photon alignment.

The magnitude of the CDAD spectrum is only about $25 \%$ that of the ARPES spectra. However, in Ref. 18 we showed that the magnitude of the CDAD spectrum for photoionization from an oriented oxygen $p$ orbital was about $50 \%$ that of the ARPES spectra. The reason for this difference in relative magnitude is threefold:

(1) As discussed in Ref. 18, CDAD from atoms arises solely from interference between the $l \rightarrow l+1$ and $l \rightarrow l-1$ photoionization channels. Thus, while the magnitude of the ARPES spectra depend roughly on $|\langle p|r| k d\rangle|^{2}$, the CDAD spectra depend on $|\langle p|r| k s\rangle| \cdot|\langle p|r| k d\rangle|$. By itself, this fact actually makes the relative magnitude of the $O$ atom CDAD spectrum about $75 \%$ that of Cs.

(2) CDAD from atoms depends on $\sin \left(\delta_{l+1}-\delta_{l-1}\right)$ where $\delta_{l+1}, \delta_{l-1}$ are the phase shifts for the $l+1$ and $l-1$ photoionization channels, respectively. ${ }^{5}$ This fact favors the

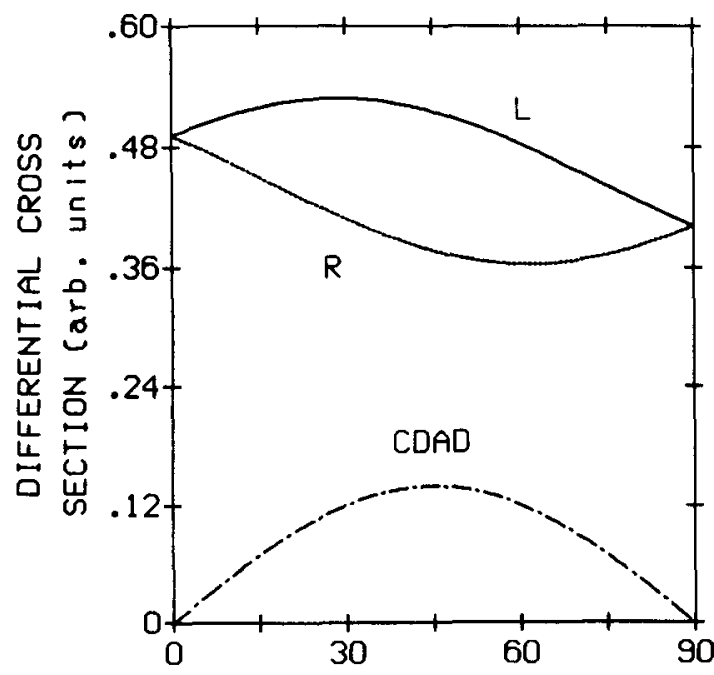

COLLECTION ANGLE $\theta_{k}$ (deg)

FIG. 5. Differential cross section vs collection angle $\theta_{k}$ for left $(L)$ and right $(R)$ circularly polarized light and CDAD from the $7 P_{3 / 2}$ state of Cs. Alignment created by one-photon absorption $6 S_{1 / 2} \rightarrow 7 P_{3 / 2}$. magnitude of the $O$ atom $C D A D$ spectrum over that for Cs by a factor of $\sim 2$.

(3) The Cs calculation incorporates spin-orbit coupling. The $P_{3 / 2}$ state is actually a linear combination of $(J, M)=(1,0)$ and $(1,1)$. Photoionization from a $(1,0)$ state has a $\sin 2 \theta_{k}$ dependence, while photoionization from a $(1,1)$ state has the same dependence, but opposite in sign and half the magnitude. ${ }^{8}$ This result is a consequence of the fact that CDAD from an isotropic distribution of states, i.e., equal population of $(1,0),(1,1)$, and $(1-1)$, must vanish ${ }^{18}$ and that CDAD spectra for photoionization from the $(1,1)$ and $(1-1)$ states are identical. The net result favors the magnitude of the $\mathrm{O}$ atom CDAD spectrum over that for $\mathrm{Cs}$ by $4 / 3$.

The three abovementioned factors give rise to the overall factor of 2 between the relative magnitudes of the CDAD spectrum for $\mathrm{O}$ atom and $\mathrm{Cs}$ when compared to their corresponding ARPES spectra.

As a final note, we point out that the $15 \%-25 \%$ magnitude of all the CDAD spectra shown should not be discouraging. For alignment created by photoabsorption or photofragmentation in which the polarization of the light defines the laboratory frame of the experiment, only the polarization vector must be rotated to vary $\theta_{k}$-the electron detector need not be moved. In addition, CDAD must vanish for symmetry reasons at $\theta_{k}=0$ and $\theta_{k}=90^{17,18}$ and, therefore, the ARPES spectra at these angles for left and right circularly polarized light must be equal. This should make normalization of the left and right ARPES spectra convenient.

\section{CONCLUSIONS}

We have demonstrated that CDAD can be used to probe alignment of atomic and molecular states in the gas phase. CDAD exists in the electric dipole approximation without the necessity of spin-orbit coupling. Although we used multiphoton absorption to create alignment, CDAD should be useful in probing alignment created by other means as well. Our results indicate that CDAD is about $15 \%-25 \%$ of ARPES for photoionization of the $A^{2} \Sigma^{+}$state of NO and for photoionization of the $7 P_{3 / 2}$ state of cesium. We believe that the estimated $15 \%-25 \%$ magnitude of CDAD makes 
these measurements feasible. Because the ARPES spectra for right and left circularly polarized light can be normalized at $\theta_{k}=0^{\circ}$ and $\theta_{k}=90^{\circ}$ (where CDAD must vanish by symmetry), ${ }^{17,18}$ some of the uncertainties in the experimental data could be eliminated.

\section{ACKNOWLEDGMENTS}

This material is based upon research supported by the National Science Foundation Under Grant No. CHE8218166. The research reported in this paper made use of the Dreyfus-NSF Theoretical Chemistry Computer which was funded through grants from the Camille and Henry Dreyfus Foundation, the National Science Foundation (Grant No. CHE-7820235), and the Sloan Fund of the California Institute of Technology. One of us (R.L.D.) gratefully acknowledges support through a National Science Foundation Graduate Fellowship.

'See, for example, E. Marinero, C. T. Rettner, and R. N. Zare, J. Chem. Phys. 80, 4142 (1984).

${ }^{2}$ See, for example, R. Vasudev, R. N. Zare, and R. N. Dixon, J. Chem. Phys. 80, 4863 (1984); I. Nadler, J. Pfab, H. Reisler, and C. Wittig, ibid. 81, 653 (1984); S. Buelow, G. Radhakrishnan, J. Catanzarite, and C. Wittig, ibid. 83, 444 (1985).

${ }^{3}$ See, for example, A. R. Burns, Phys. Rev. Lett. 55, 525 (1985).

'See, for example, G. M. McClelland, G. D. Kubiak, H. G. Rennagel, and R. N. Zare, Phys. Rev. Lett. 46, 831 (1981); F. Frenkel, J. Häger, H. Krieger, H. Walther, C. T. Campbell, G. Ertl, H. Kuipers, and J. Segner, ibid. 46, 152 (1981).

${ }^{5}$ W. Jitschin, S. Osimitsch, H. Reihl, H. Kleinpoppen, and H. O. Lutz, J.
Phys. B 17, 1899 (1984).

${ }^{6}$ P. Franken, K. Sands, and J. Hobart, Phys. Rev. Lett. 1, 118 (1958); R. Novick and H. E. Peters, ibid. 1, 54 (1958).

${ }^{7}$ D. H. Parker, K. K. Chakravorty, and R. B. Bernstein, J. Phys. Chem. 85, 466 (1981).

${ }^{8}$ E. D. Poliakoff, J. L. Dehmer, D. Dill, A. C. Parr, K. H. Jackson, and R. N. Zare, Phys. Rev. Lett. 46, 907 (1981)

${ }^{9}$ (a) C. H. Greene and R. N. Zare, Annu. Rev. Phys. Chem. 33, 119 (1982); J. Chem. Phys. 78, 6741 (1983); (b) R. E. Drullinger and R. N. Zare, ibid. 59, 4225 (1973).

${ }^{10}$ R. N. Zare, Ber. Bunsenges. Phys. Chem. 86, 422 (1982).

"S. N. Dixit and V. McKoy, J. Chem. Phys. 82, 3546 (1985).

${ }^{12}$ M. G. White, W. A. Chupka, M. Seaver, A. Woodward, and S. D. Colson, J. Chem. Phys. 80, 678 (1984); W. J. Kessler and E. D. Poliakoff, ibid. 84, 3647 (1986).

${ }^{13}$ See, for example, L. Velluz, M. Legrand, and M. Grosjean, Optical Circular Dichroism (Academic, New York, 1965).

${ }^{14}$ B. Ritchie, Phys. Rev. A 13, 1411 (1976); 14, 359 (1976); 14, 1396 (1976).

${ }^{15}$ N. A. Cherepkov, J. Phys. B 16, 1543 (1983).

${ }^{16}$ E. A. Power and T. Thirunamachandran, J. Chem. Phys. 60, 3695 (1974); E. A. Power, ibid. 63, 1348 (1975).

${ }^{17}$ R. L. Dubs, S. N. Dixit, and V. McKoy, Phys. Rev. Lett. 54, 1249 (1985).

${ }^{18}$ R. L. Dubs, S. N. Dixit, and V. McKoy, Phys. Rev. B 32, 8389 (1985).

${ }^{19}$ B. Ritchie, Phys. Rev. A 12, 567 (1975).

${ }^{20}$ R. Parzynski, Act. Phys. Pol. A 57, 49 (1980)

${ }^{21}$ R. R. Lucchese, G. Raseev, and V. McKoy, Phys. Rev. A 25, 2572 (1982).

${ }^{22}$ S. N. Dixit, D. L. Lynch, V. McKoy, and W. M. Huo, Phys. Rev. A 32, 1267 (1985).

${ }^{23}$ M. E. Rose, Elementary Theory of Angular Momentum (Wiley, New York, 1957).

${ }^{24}$ S. N. Dixit and P. Lambropoulos, Phys. Rev. A 27, 861 (1983).

${ }^{25}$ J. C. Tully, R. S. Berry, and B. J. Dalton, Phys. Rev. 176, 95 (1968).

${ }^{26}$ P. J. Brussard and H. A. Tolhoek, Physica 23, 955 (1957); R. N. Zare (unpublished). 